\title{
Cómo puede evaluar el patólogo si la exéresis del meso-recto y del carcinoma rectal ha sido adecuada
}

\author{
F. Domínguez Iglesias
}

La resección quirúrgica continúa siendo la aproximación estándar al tratamiento primario del cáncer de recto. Aproximadamente el $84 \%$ de los pacientes con este tipo de neoplasia experimentan cirugía como modalidad primaria de tratamiento con intento curativo, escisión completa en la mayoría de los casos. Sin embargo, las recurrencias locales tras cirugía curativa son un problema clínico importante, que afecta de manera muy grave a la calidad de vida de los pacientes y frecuentemente desemboca en la muerte. Varios estudios previos han sugerido que la causa principal que provoca recurrencias locales es el fallo para lograr una remoción completa del tumor ${ }^{1}$. En los últimos años se han producido dos cambios importantes para el tratamiento del cáncer de recto. La modificación del tratamiento quirúrgico y la escisión meso-rectal total (EMT), aparece como un procedimiento de elección determinando una reducción de los ratios de recurrencia local del $30-40 \%$ al 5-15\%2. La técnica se basa en la remoción del tumor junto con la columna de grasa mesorrectal, para minimizar la carga de tumor residual. El papel del cirujano y su experiencia en la cirugía del cáncer rectal aparece aquí como figura central para el tratamiento primario de la enfermedad ${ }^{3}, 4$. De otra parte, la radioterapia preoperatoria ha jugado un papel importante disminuyendo las recurrencias locales del $27 \%$ al $11 \%$.

En este contexto la evaluación patológica de los especímenes de resección por cáncer de recto aparece como un factor clave, ya que puede aportar datos de la mayor relevancia para el manejo postoperatorio de los pacientes, establecer parámetros pronósticos y validar la calidad de la cirugía realizada. Los elementos fundamentales que aporta el estudio patológico de estos especímenes incluyen el tipo de tumor, grado histológico, estatus de márgenes de resección quirúrgicos, presencia de invasión vascular y determinación del estadio patológico de la enfermedad ${ }^{\text {. El }}$ El objetivo de la presente revisión es exponer el papel del patólogo en la eva-

Servicio de Anatomía Patológica

Hospital Valle del Nalón

Langreo (Asturias) luación de la calidad de la exéresis quirúrgica con EMT del cáncer de recto y la adecuación de los márgenes.

\section{Evaluación patológica de la resección meso-rectal}

Aunque pueden persistir controversias acerca del valor de este procedimiento técnico, hay evidencias de que la EMT puede representar el más importante determinante técnico para el correcto manejo quirúrgico del cáncer de recto, atendiendo a sus dos objetivos principales que son la resección tumoral completa y la preservación funcional. Descrita por Heald et al. ${ }^{2}$, la técnica de EMT consiste en la disección precisa y cuidadosa en la pelvis alrededor del recto, tratando de preservar la integridad de la fascia visceral rectal, que representa la parte más externa de la cubierta de tejido adiposo que lo envuelve circunferencialmente, y de la fascia parietal que recubre la parte anterior del sacro y de la pelvis late$\mathrm{ral}^{2,7}$. En estos casos, la calidad de la cirugía puede ser valorada en gran medida por la calidad del espécimen de resección. La evaluación patológica del grado de integridad de la superficie meso-rectal se ha visto que correlaciona clínicamente con la probabilidad de recurrencia local y a distancia y con la afectación del margen radial circunferencial (MRC). De manera concreta, un meso-recto macroscópicamente incompleto predice un alto ratio de afectación del MRC y de recurrencias locales, en comparación con una escisión completa o casi completa ${ }^{8,9}$. Un meso-recto incompleto es definido como aquel que tiene poca masa y defectos macroscópicos hasta la muscularis propria y/o una superficie circunferencial muy irregular. Por el contrario, en la resección completa o casi completa la masa meso-rectal aparece grande o moderada, con irregularidades sólo menores en la superficie meso-rectal 6,9 (Tabla I). Nagtegaal et al ${ }^{9}$ observaron que los pacientes con resección incompleta así definida tenían un riesgo de recurrencia local y a distancia de la enfermedad del $36,1 \%$, frente a un $20,3 \%$ en el grupo con un meso-recto completo. De esta manera, la calidad macroscópica del espécimen de resección rectal, juzgado por la cantidad de tejidos blandos extramurales en la envoltura meso-rectal y el grado de integridad de la fascia meso-rectal, refleja directamente la calidad de la técnica quirúrgica y se correlaciona con predictores clínicamente relevantes del desenlace de la enfermedad 8 -10. 


\section{F. Domínguez Iglesias}

TABLA I

\section{Evaluación macroscópica de especímenes de escisión meso-rectal total ${ }^{6}$}

- Completa

Masa meso-rectal intacta con superficie lisa.

Irregularidades menores de la superficie meso-rectal.

Ningún defecto de superficie mayor de $5 \mathrm{~mm}$ de profundidad.

No estrechamiento hacia el margen distal del espécimen.

En la sección transversa el margen circunferencial aparece liso.

- Casi completa

Masa meso-rectal moderada.

Irregularidades de la superficie mesorrectal con defectos mayores de $5 \mathrm{~mm}$ pero ninguno llegando a la muscularis propia.

Muscularis propria no visible en ningún sitio, con excepción de la inserción de músculos levator ani.

Moderado estrechamiento del espécimen hacia el margen distal.

- Incompleta

Masa meso-rectal muy escasa.

Defectos en el meso-recto hasta la muscularis propria

En secciones transversas el margen circunferencial aparece muy irregular.

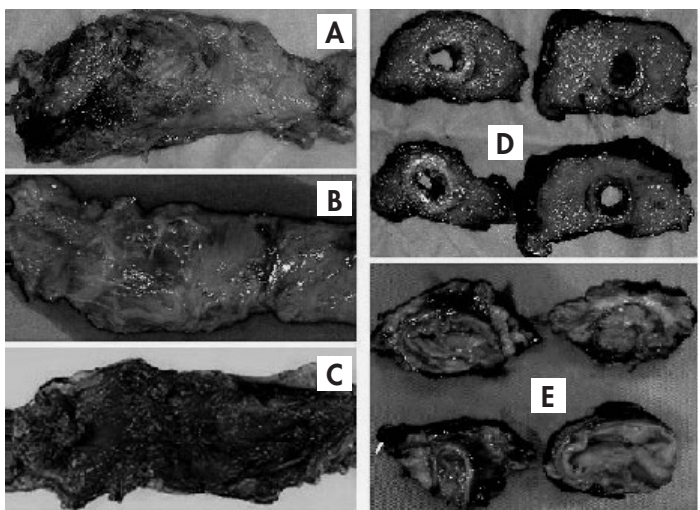

Fig. 1. Ilustraciones de las definiciones dadas en la tabla 1. (A y D) Meso-recto completo, sin (A) defectos y con (D) margen circunferencial liso. ( $B$ y E) Resección casi completa, con (B) moderada masa de meso-recto perirrectal y $(E)$ irregularidad en el margen circunferencial. (C) meso-recto incompleto con defectos profundos.

El examen patológico estandarizado de las resecciones es llevado a cabo en nuestro laboratorio de acuerdo con el protocolo descrito por Quirke et al. ${ }^{11}$ y Nagtegaal et al. ${ }^{9}$. A su llegada al laboratorio, el espécimen de resección es valorado para la integridad de la EMT, de acuerdo con las definiciones descritas, luego se toman fotografías del mismo y tras la fijación y marcado de bordes quirúrgicos con tinta indeleble se realizan secciones coronales que son adicionalmente fotografiadas (Fig. 1). Se realiza un cuidadoso examen del MRC y del nivel de invasión tumoral en la pared intestinal y tejidos que la rodean, se toman medidas de dos diámetros del tumor y de la profundidad de invasión en milímetros y se aíslan y se procesan para estudio histológico todos los ganglios extraídos, así como al menos cuatro muestras del tumor, pared no tumoral, lesiones asociadas y bordes transversales. La documentación fotográfica de estos especímenes es de especial interés por su aspecto docente, dado que el entrenamiento de cirujanos y patólogos se ha visto que influye en la calidad de la técnica quirúrgica y en su evaluación correcta $^{3,10}$

\section{La cuestión de los márgenes quirúrgicos}

Los márgenes que requieren evaluación en las resecciones por cáncer rectal son el margen proximal, distal y el margen mesentérico o margen radial circunferencial (MRC). En nuestro laboratorio, los márgenes distal y proximal de las escisiones rectales son estudiados histológicamente en secciones transversales $y$, si es posible, con una sección adicional, longitudinal, que demuestre la relación del tumor con el margen más próximo. Cuando el tumor se localiza a más de $50 \mathrm{~mm}$ del margen se ha sugerido que no es necesario el examen histológico, pues la probabilidad de recurrencias anastomóticas es muy baja ${ }^{12}$. En los especímenes de resección rectal anterior, sin embargo, el margen distal es frecuentemente crítico por los problemas inherentes a la propia cirugía. Se considera que un margen de $2 \mathrm{~cm}$ de tejido normal puede ser correcto para prevenir recurrencias locales, e incluso en tumores T1 y T2 márgenes de $\leq 10 \mathrm{~mm}$ pueden ser suficientes $^{13}$. Cuando el tumor está a más de $30 \mathrm{~mm}$ del margen en el espécimen principal, los anillos quirúrgicos con grapas pueden no ser estudiados histológicamente ${ }^{14}$.

El MRC en el cáncer de recto es definido como el margen de tejidos blandos adventiciales o perineales más próximos al punto de penetración máxima del tumor. Numerosos estudios han probado que, más bien que los márgenes mucosos, el MRC es un elemento clave para predecir las recurrencias locales, a distancia y la supervivencia global de los pacientes, incluidos aquellos que experimentan EMT11, 13, 15, 16. Nagtegaal et al. ${ }^{16}$ mostraron que en pacientes que experimentan cirugía con EMT por cáncer rectal un $M R C \leq 2 \mathrm{~mm}$ se asociaba a un riesgo de recurrencia local del $16 \%$, mientras que pacientes con MRC $\leq 1 \mathrm{~mm}$ tenían un riesgo incrementado de metástasis a distancia y períodos más cortos de supervivencia. El valor pronóstico del MRC fue independiente del estadio TNM. Pese a estos datos, el informe del MRC no es 
práctica de rutina en muchos laboratorios. Un estudio demostró que el MRC fue evaluado patológicamente en sólo $21 \%$ de los casos ${ }^{17}$.

La evaluación patológica del MRC fue descrita por Quirke et al. ${ }^{11}$ y más recientemente por Nagtegaal et al. ${ }^{16}$. Las piezas de resección estarán bien fijadas y marcados con tinta indeleble sus bordes quirúrgicos. El espécimen será fijado abierto, excepto en la zona del tumor para preservar la anatomía rectal. Tras la fijación el espécimen será seccionado transversalmente en secciones coronales múltiples a través del tumor y mesorrecto. El MCR macroscópico es medido con una regla. Se tomarán el número necesario de bloques del tumor primario y de ganglios linfáticos en relación con el MRC $y$, cuando el tumor o ganglios sospechosos se aproximan al margen (por ejemplo distancia $<1 \mathrm{~mm}$ ), las medidas se repetirán microscópicamente y se informarán con independencia de la proximidad del tumor al borde. Una distancia del tumor o metástasis ganglionar del mismo al MRC $\leq 1$ $\mathrm{mm}$ será considerada como afectación del borde ${ }^{16}$. La gran relevancia clínica de la afectación tumoral o la proximidad del MRC enfatiza la necesidad de una cuidadosa evaluación patológica y el informe del estatus microscópico de MCR en todos los especímenes de resección por cáncer de recto.

Como conclusión, podemos decir que el estudio meticuloso, tanto macroscópico como microscópico, de las piezas de resección por cáncer rectal es fundamental para la evaluación de la calidad quirúrgica de la intervención y aporta información de notable relevancia para el manejo posterior y aproximación al pronóstico de los pacientes con estas neoplasias.

\section{Bibliografía}

1. Mc Ardle CS, Hole D. Impact of variability among surgeons on postoperative morbidity and mortality and ultimate survival. BMJ 1991; 302:1501-5.

2. Heald RJ, Ryall RDH. Recurrence and survival after total mesorectal escisión for rectal cancer. Lancet 1986; 1:1479-82.

3. Hermanek P, Mansmann U, Staimer D, et al. The German experience: The surgeon as a prognostic factor in colon and rectal cancer surgery. Surg Oncol Clin North Am 2000; 9:3349.

4. Lehander Martling A, Holm T, Rutqvist LE, Moran BJ, Heald RJ,
Cedermark B. Effect of a surgical training programme on outcome of rectal cancer in the county of Stockholm. Lancet 2000; 356:93-6.

5. Kapiteijn E, Marijnen CAM, Nagtegaal ID, et al. Preoperative radiotherapy combined with total mesorectal excision for respectable rectal cancer. N Engl J Med 2001; 345:638-46.

6. Compton CC. Colorectal carcinoma: diagnostic, prognostic, and molecular features. Mod Pathol 2003; 16:376-88.

7. Heald RJ Moran BJ, Ryall RD, Sexton R, Mcfarlane JK. Rectal cancer. The Basingstoke experience of total mesorectal excision, 1978-1997. Arch Surg 1998; 133:894-9.

8. Hermanek P, Hohenberger W: The importance of volume in colorectal cancer surgery. Eur J Surg Cancer 2000; 22: 213-5.

9. Nagtegaal ID, Cornelis JH, van der Worp E, et al. Macroscopic evaluation of rectal cancer resection specimen: clinical significance of the pathologist in quality control. J Clin Oncol 2002; 20:1729-34

10. Nagtegaal ID, van Krieken JH. The role of pathologists in the quality control of diagnosis and treatment of rectal cancer. An overview. Eur J Cancer 2002; 38:964-72.

11. Quirke P, Durdey P, Dixon MF, Williams NS. Local recurrence of rectal adenocarcinoma due to inadequate surgical resection. Lancet 1986; 2:996-9.

12. Cross SS, Bull AD Smith JHF. Is there any justification for the routine examination of bowel resection margins in colorectal adenocarcinoma? J Clin Pathol 1989; 42:1040-2.

13. Quirke P, Scott N. The pathologist's role in the assessment of local recurrence in rectal carcinoma. Surg Oncol Clin N Am 1992; 3:1-17.

14. Sloane JP, Ansell ID, Quirke P, Underwood JCE. Standards and minimum datasets for reporting common cancers. Minimum dataset for colorectal cancer histopathology reports. London: The Royal College of Pathologists; 1998.

15. Adam IJ, Mohamdee MO, Martin IG, et al. Role of circumferential margin involvement in the local recurrence of rectal cancer. Lancet 1994; 344:707-11.

16. Nagtegaal ID, Marijnen CAM, Kranenbarg EK, van de Velde $\mathrm{CJH}$, krieken JHJM. Circumferential margin involvement is still an important predictor of local recurrence in rectal carcinoma. Am J Surg Pathol 2002; 26:350-7.

17. Stacchi L, Nelson H, Sargent DJ, et al. Impact of surgical and pathological variables in rectal cancer: a United States community and cooperative group report. J Clin Oncol 2001; 19:3895-902.
Correspondencia:

Dr. F. Domínguez Iglesias Servicio de Anatomía Patológica Hospital Valle del Nalón Polígono de Riaño, s/n E-33920 Langreo (Asturias) E-mail: fdoig59@yahoo.es 